\title{
STOCHASTIC SOLUTIONS FOR THE TWO-DIMENSIONAL ADVECTION-DIFFUSION EQUATION*
}

\author{
$\mathrm{XIAOLIANG} \mathrm{WAN}^{\dagger}$, DONGBIN XIU ${ }^{\dagger}$, AND GEORGE EM KARNIADAKIS ${ }^{\dagger}$
}

\begin{abstract}
In this paper, we solve the two-dimensional advection-diffusion equation with random transport velocity. The generalized polynomial chaos expansion is employed to discretize the equation in random space while the spectral $/ h p$ element method is used for spatial discretization. Numerical results which demonstrate the convergence of generalized polynomial chaos are presented. Specifically, it appears that the fast convergence rate in the variance is the same as that of the mean solution in the Jacobi-chaos unlike the Hermite-chaos. To this end, a new model to represent compact Gaussian distributions is also proposed.
\end{abstract}

Key words. generalized polynomial chaos, advection-diffusion, stochastic modeling

AMS subject classifications. $65 \mathrm{C} 20,65 \mathrm{C} 30$

DOI. $10.1137 / \mathrm{S} 106482750342684 \mathrm{X}$

1. Introduction. The importance of uncertainty modeling is clearly recognized in scientific computing, and there has been a growing interest in applications of probabilistic methods [5, 11, 13, 18]. Among the existing methods, polynomial chaos expansion has been shown to work effectively for many problems. It is based on the homogeneous chaos theory of Wiener [23] and has been applied to various practical applications by Ghanem and coworkers; see, e.g., [7, 8, 9, 10, 15]. The classical polynomial chaos expansion employs Hermite orthogonal polynomials in terms of Gaussian random variables to represent stochastic processes and is essentially a spectral expansion of random variables. Cameron and Martin have proven that this expansion converges to any $L_{2}$ functionals in the random space in the mean-square sense [3]. Combined with Karhunen-Loeve decomposition of the stochastic inputs [16], polynomial chaos results in computationally tractable algorithms for large engineering systems. Other applications and analysis, including the limitation of Wiener-Hermite expansion, can be found in $[4,17,20]$.

More recently, a more general framework, called the generalized polynomial chaos, was developed [26], following the more fundamental work on stochastic theory [19, 22] and orthogonal polynomials $[1,14]$. The generalized polynomial chaos employs new classes of orthogonal polynomials and is more efficient to represent general nonGaussian processes. Applications to stochastic ODEs, PDEs, Navier-Stokes equations, and flow-structure interactions have been reported, along with convergence for model problems in [25, 26, 27].

In this paper, we consider the two-dimensional advection-diffusion equation with random transport velocity. This can be considered as a model of transport phenomena in random media, which has been a subject of intensive research; see, for example, $[21,24]$. In this paper, we use this equation as a means of examining the approximation properties of generalized polynomial chaos. Different types of random distributions are considered, and convergence rate is examined using exact solutions.

\footnotetext{
* Received by the editors April 30, 2003; accepted for publication (in revised form) April 9, 2004; published electronically December 22, 2004. This work was supported by DOE, AFOSR, and NSF. http://www.siam.org/journals/sisc/26-2/42684.html

${ }^{\dagger}$ Division of Applied Mathematics, Brown University, Providence, RI 02912 (xlwan@dam.brown. edu,xiu@dam.brown.edu,gk@dam.brown.edu).
} 
This paper is organized as follows. In section 2, we briefly review the concept of generalized polynomial chaos. The formulation of stochastic advection-diffusion equation and its chaos expansion are presented in section 3. Numerical results are in section 4 , and we conclude the paper with a short summary in section 5 .

2. Generalized polynomial chaos. The generalized polynomial chaos is a means of representing second-order stochastic processes $X(t, x, \omega)$, viewed as a function of $\omega$ (the random event) and $t, x$ (variables in time and space, respectively):

$$
\begin{aligned}
X(t, x, \omega)= & a_{0} \Psi_{0} \\
& +\sum_{i_{1}=1}^{\infty} a_{i_{1}} \Psi_{1}\left(\xi_{i_{1}}(\omega)\right) \\
& +\sum_{i_{1}=1}^{\infty} \sum_{i_{2}=1}^{i_{1}} a_{i_{1} i_{2}} \Psi_{2}\left(\xi_{i_{1}}(\omega), \xi_{i_{2}}(\omega)\right) \\
& +\sum_{i_{1}=1}^{\infty} \sum_{i_{2}=1}^{i_{1}} \sum_{i_{3}=1}^{i_{2}} a_{i_{1} i_{2} i_{3}} \Psi_{3}\left(\xi_{i_{1}}(\omega), \xi_{i_{2}}(\omega), \xi_{i_{3}}(\omega)\right) \\
& +\cdots,
\end{aligned}
$$

where $\Psi_{n}\left(\xi_{i_{1}}, \ldots, \xi_{i_{n}}\right)$ denotes the generalized polynomial chaos of order $n$ in the variables $\left(\xi_{i_{1}}, \ldots, \xi_{i_{n}}\right)$ and are orthogonal polynomials in terms of the multidimensional random variables $\boldsymbol{\xi}=\left(\xi_{i_{1}}, \ldots, \xi_{i_{n}}\right)$. For notational convenience, one can rearrange the terms in (2.1), according to some numbering scheme, and rewrite the expansion as

$$
X(x, t, \omega)=\sum_{j=0}^{\infty} \hat{a}_{j}(x, t) \Phi_{j}(\boldsymbol{\xi}),
$$

where there is a one-to-one correspondence between the functions $\Psi_{n}\left(\xi_{i_{1}}, \ldots, \xi_{i_{n}}\right)$ and $\Phi_{j}(\boldsymbol{\xi})$, and their corresponding coefficients $a_{i_{1} i_{2} i_{3} \ldots}$ and $\hat{a}_{j}$. Again $\left\{\Phi_{j}(\boldsymbol{\xi})\right\}$ are the (multidimensional) orthogonal polynomials in terms of the multidimensional random vector $\boldsymbol{\xi}$, satisfying the orthogonality relation

$$
\left\langle\Phi_{i}, \Phi_{j}\right\rangle=\left\langle\Phi_{i}^{2}\right\rangle \delta_{i j}
$$

where $\delta_{i j}$ is the Kronecker delta and \langle\rangle denotes the ensemble average. This is the inner product in the Hilbert space determined by the probability measure of the random variables

$$
\langle f(\boldsymbol{\xi}), g(\boldsymbol{\xi})\rangle=\int f(\boldsymbol{\xi}) g(\boldsymbol{\xi}) w(\boldsymbol{\xi}) d \boldsymbol{\xi}
$$

with $w(\boldsymbol{\xi})$ denoting the weighting function. In the discrete case, the above orthogonal relation takes the form

$$
\langle f(\boldsymbol{\xi}), g(\boldsymbol{\xi})\rangle=\sum_{\boldsymbol{\xi}} f(\boldsymbol{\xi}) g(\boldsymbol{\xi}) w(\boldsymbol{\xi}) .
$$

Note that the above integral and summation are taken in the domain defined by the support of the underlying random variables $\boldsymbol{\xi}$, and the summation in (2.5) is taken over all possible values of $\boldsymbol{\xi}$. 
TABle 2.1

Correspondence of the types of Wiener-Askey polynomial chaos and their underlying random variables $(N \geq 0$ is a finite integer).

\begin{tabular}{|c|c|c|c|}
\hline & Random variables $\boldsymbol{\xi}$ & Wiener-Askey chaos $\{\Phi(\boldsymbol{\xi})\}$ & Support \\
\hline \hline Continuous & Gaussian & Hermite-chaos & $(-\infty, \infty)$ \\
& gamma & Laguerre-chaos & {$[0, \infty)$} \\
& beta & Jacobi-chaos & {$[a, b]$} \\
& uniform & Legendre-chaos & {$[a, b]$} \\
\hline Discrete & Poisson & Charlier-chaos & $\{0,1,2, \ldots\}$ \\
& binomial & Krawtchouk-chaos & $\{0,1, \ldots, N\}$ \\
& negative binomial & Meixner-chaos & $\{0,1,2, \ldots\}$ \\
& hypergeometric & Hahn-chaos & $\{0,1, \ldots, N\}$ \\
\hline
\end{tabular}

In (2.2), there is a one-to-one correspondence between the type of the orthogonal polynomials $\{\Phi\}$ and the type of the random variables $\boldsymbol{\xi}$. This is determined by choosing the type of the orthogonal polynomials $\{\Phi\}$ in such a way that their weighting function $w(\boldsymbol{\xi})$ in the orthogonality relation of (2.4) has the same form as the probability distribution function of the underlying random variables $\boldsymbol{\xi}$. For example, the weighting function of $n$-dimensional Hermite orthogonal polynomials is $\frac{1}{\left((2 \pi)^{n}\right)^{1 / 2}} \exp \left(-\frac{1}{2} \boldsymbol{\xi}^{T} \boldsymbol{\xi}\right)$ and is the same as the probability density function of the $n$ dimensional Gaussian random variables $\boldsymbol{\xi}$. Hence, the classical Wiener polynomial chaos is an expansion of Hermite polynomials in terms of Gaussian random variables. Some types of generalized polynomial chaos corresponding to the commonly known distributions are listed in Table 2.1.

The expansion of (2.1) (or (2.2)) resides in an infinite-dimensional space determined by $\boldsymbol{\xi}$ and is an infinite summation. In practice, we have to restrict ourselves to the finite-term summation. This is achieved by reducing the expansion to a finitedimensional space, i.e., expansion of finite-dimensional random variables $\boldsymbol{\xi}$, according to the nature of random inputs; we also set the highest order of the polynomials $\{\Phi\}$ according to accuracy requirement. The finite-term expansion takes the form

$$
X(\omega)=\sum_{j=0}^{M} \hat{a}_{j} \Phi_{j}(\boldsymbol{\xi}),
$$

where $\boldsymbol{\xi}$ is an $n$-dimensional random vector. Multidimensional generalized polynomial bases are constructed as the tensor products of the corresponding one-dimensional bases.

3. Stochastic advection-diffusion equation. In this paper, we consider the two-dimensional advection-diffusion equation with random transport velocity,

$$
\frac{\partial \phi}{\partial t}(\boldsymbol{x}, t ; \omega)+\boldsymbol{u}(\boldsymbol{x} ; \omega) \cdot \nabla \phi=\nu \nabla^{2} \phi \quad(\boldsymbol{x}, t ; \omega) \in D \times R^{+} \times \Omega,
$$

where $D$ is a bounded domain in $R^{2}, \Omega$ is the sample space in an appropriately defined probability space, and $\nu$ is the viscosity. In this paper, we will assume deterministic boundary and initial conditions. The transport velocity field is $\boldsymbol{u}(\boldsymbol{x} ; \omega)=$ $u(x, y ; \omega) \boldsymbol{e}_{x}+v(x, y ; \omega) \boldsymbol{e}_{y}$, and we will focus on large-scale random perturbations; i.e., the random field is strongly correlated and retains certain smoothness. The problem of stochastic advection-diffusion subject to white noise input will not be considered here. 
By using the generalized polynomial chaos expansion of (2.6), we can expand the solution process and the transport velocity field as

$$
\phi(\boldsymbol{x}, t ; \omega)=\sum_{i=0}^{M} \phi_{i}(\boldsymbol{x}, t) \Phi_{i}(\boldsymbol{\xi}(\omega)), \quad \boldsymbol{u}(\boldsymbol{x} ; \omega)=\sum_{i=0}^{M} \boldsymbol{u}_{i}(\boldsymbol{x}) \Phi_{i}(\boldsymbol{\xi}(\omega)) .
$$

By substituting these expansions into governing equation (3.1), we obtain

$$
\sum_{i=0}^{M} \frac{\partial \phi_{i}}{\partial t} \Phi_{i}+\sum_{i=0}^{M} \sum_{j=0}^{M} \boldsymbol{u}_{i} \cdot \nabla \phi_{j} \Phi_{i} \Phi_{j}=\nu \sum_{i=0}^{M} \nabla^{2} \phi_{i} \Phi_{i}
$$

A Galerkin projection of the above equation onto each polynomial basis $\left\{\Phi_{i}\right\}$ is then conducted to ensure the error is orthogonal to the functional space spanned by the finite-dimensional basis $\left\{\Phi_{i}\right\}$. By projection with $\Phi_{k}$ for each $k=\{0, \ldots, M\}$ and employing the orthogonality relation of $(2.3)$, we obtain for each $k=0, \ldots, M$,

$$
\frac{\partial \phi_{k}}{\partial t}+\frac{1}{\left\langle\Phi_{k}^{2}\right\rangle} \sum_{i=0}^{M} \sum_{j=0}^{M} \boldsymbol{u}_{i} \cdot \nabla \phi_{j} e_{i j k}=\nu \nabla^{2} \phi_{k}
$$

where $e_{i j k}=\left\langle\Phi_{i} \Phi_{j} \Phi_{k}\right\rangle$. Together with $\left\langle\Phi_{k}^{2}\right\rangle$, these coefficients can be evaluated analytically from the definition of the polynomial basis $\left\{\Phi_{i}\right\}$.

Equation (3.4) is a set of $(M+1)$ PDEs coupled through the advection terms. The total number of expansion terms is determined by the dimensionality of random space $(n)$ and the highest order of the polynomial expansion $(p)$ from

$$
(M+1)=\frac{(n+p) !}{n ! p !} .
$$

The equations obtained from (3.4) are deterministic and can be solved readily by any conventional numerical schemes. In this paper, we employ the spectral $/ h p$ element method to obtain high accurate results in physical space [12]. A second-order stiffly stable scheme, also called backward differentiation scheme, is employed for the timeintegration (cf. [6]).

4. Numerical results. In this section, we present numerical solutions of the two-dimensional advection-diffusion equation via the generalized polynomial chaos expansion.

4.1. Model problem: Convergence. We first consider a simple model problem where its exact solution is available. We assume that the transport velocity is a circular motion plus a constant random perturbation, i.e.,

$$
\boldsymbol{u}(\boldsymbol{x} ; \omega)=(y+a(\omega),-x-b(\omega)),
$$

where $a(\omega)$ and $b(\omega)$ are random variables. The initial condition is a Gaussian-shape cone,

$$
\phi(\boldsymbol{x}, 0 ; \omega)=e^{-\left[\left(x-x_{0}\right)^{2}+\left(y-y_{0}\right)^{2}\right] / 2 \lambda^{2}},
$$

and the corresponding exact stochastic solution is

$$
\phi_{e}(\boldsymbol{x}, t ; \omega)=\frac{\lambda^{2}}{\lambda^{2}+2 \nu t} e^{-\left(\hat{x}^{2}+\hat{y}^{2}\right) / 2\left(\lambda^{2}+2 \nu t\right)},
$$


where

$$
\left\{\begin{array}{l}
\hat{x}=x+b(\omega)-\left(x_{0}+b(\omega)\right) \cos t-\left(y_{0}+a(\omega)\right) \sin t \\
\hat{y}=y+a(\omega)+\left(x_{0}+b(\omega)\right) \sin t-\left(y_{0}+a(\omega)\right) \cos t .
\end{array}\right.
$$

By using the exact solution we examine the "mean-square" error of numerical solutions defined by

$$
e_{2}(\boldsymbol{x}, t)=\left(E\left[\phi_{M}(\boldsymbol{x}, t ; \omega)-\phi_{e}(\boldsymbol{x}, t ; \omega)\right]^{2}\right)^{1 / 2},
$$

where $E(\cdot)$ denotes the "expectation" operator and $\phi_{M}$ is the numerical solution obtained by $(M+1)$-term expansion. We then examine the $L_{\infty}$-norm of $e_{2}(\boldsymbol{x}, t)$ at some fixed time $t$ in the physical space. Here we set $\nu=10^{-5}, \lambda=1 / 8$, and $a(\omega)=b(\omega)=0.05 \xi$, where $\xi$ is a continuous random variable with zero mean. The final integration time is set as $t=3 \pi$, corresponding to $3 / 2$ revolutions of the Gaussian cone.

4.1.1. Beta random input and Jacobi-chaos. Here we assume that $\xi \sim$ $B e^{(\alpha, \beta)}(-1,1)$ is a beta random variable defined in $(-1,1)$ with parameters $\alpha, \beta>-1$; correspondingly, the Jacobi-chaos is employed. The result of uniform random input $(\alpha=\beta=0)$ by Legendre-chaos is shown in Figure 1. We observe that errors in both the mean and variance decrease exponentially fast as the order of chaos expansion $(p)$ increases. This is in accordance with the results in [25, 26, 27].

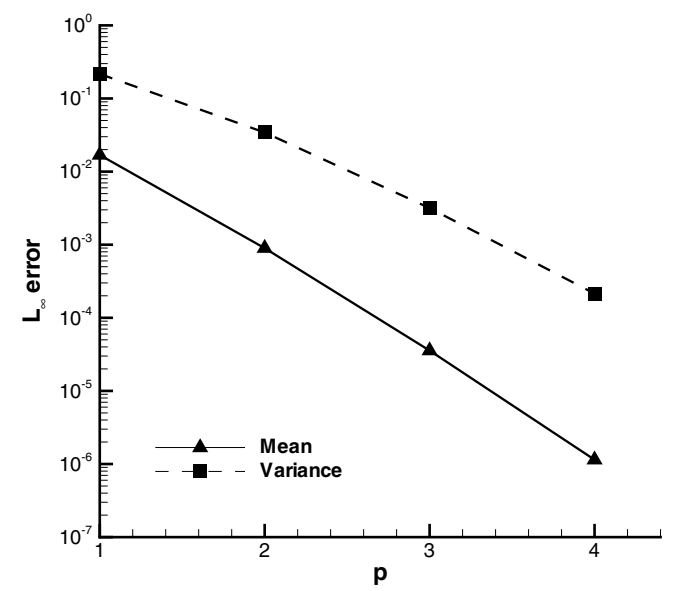

FIG. 1. $L^{\infty}$ error of Legendre-chaos with uniform random input at $T=3 \pi$.

In Figure 2, the error convergence of $B e^{(10,10)}(-1,1)$ random input is shown, and similar exponential convergence is obtained.

4.1.2. Gaussian random input and Hermite-chaos. Figure 3 shows the convergence rate of Hermite-chaos expansion when the input follows a Gaussian distribution, i.e., $\xi \sim N(0,1)$.

In Figure 4, the probability density function (PDF) of the solution at its peak location is shown at $t=\pi$, along with the corresponding PDF from the exact solution of (4.3). The peak of the solution is in the range of $\left[0, \phi_{\max }\right]$, where $\phi_{\max } \leq \frac{\lambda^{2}}{\lambda^{2}+2 \nu t} \leq 1$. 


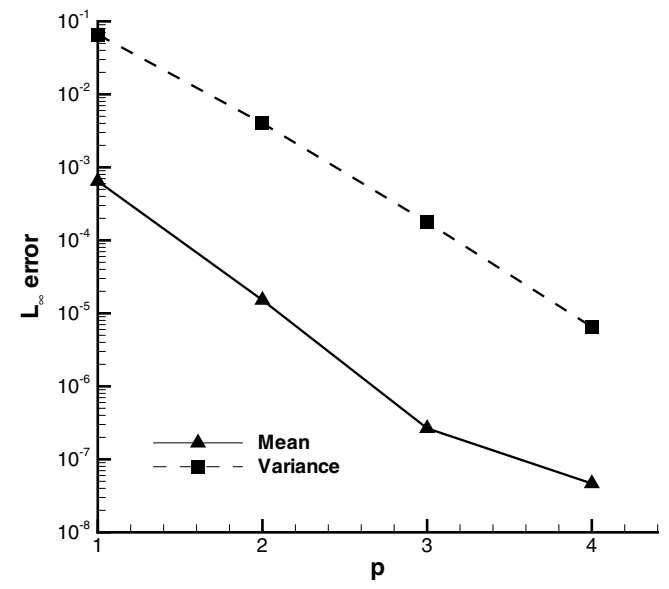

Fig. 2. $L^{\infty}$ error of Jacobi-chaos with $B e^{(10,10)}(-1,1)$ random input at $T=3 \pi$.

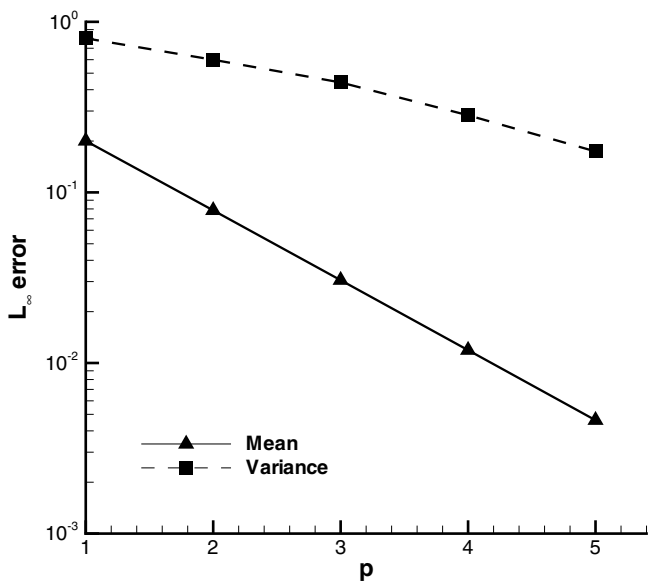

Fig. 3. $L^{\infty}$ error of Hermite-chaos with Gaussian random input at $T=3 \pi$.

Thus, its PDF should be strictly bounded on both sides, i.e., with no tails present. It is seen from Figure 4 that the Hermite-chaos approximates the exact PDF well, except from the apparent Gibb's oscillations around zero. However, the numerical PDF is clearly not bounded from below and has a thin tail along the negative axis. In fact, since Gaussian random variables have infinite support, i.e., $\xi \in(-\infty, \infty)$, we expect Hermite-chaos expansions, which are polynomial functions of Gaussian variables, to retain the infinite long tail. In this particular problem, the long tail indicates the existence of an unphysical solution with very small but nonzero probability. The difficulty of representing variables with bounded support by Hermite-chaos has also been discussed in [26]. 


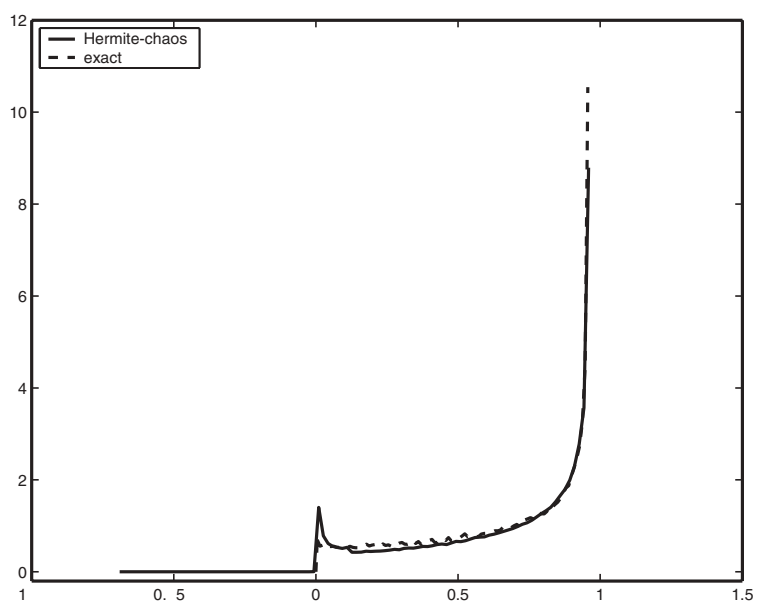

FIG. 4. PDF of the peak solution at $T=\pi$ with Gaussian input and Hermite-chaos expansion.

4.1.3. "Truncated" Gaussian input and Jacobi-chaos. An alternative to the Gaussian distribution is a "truncated" Gaussian distribution, first introduced in [28]. This is a Jacobi-chaos approximation to Gaussian distributions. It approximates Gaussian distributions closely with no long tails. It has been shown that the long tails of Gaussian distribution can result in ill-posedness of Hermite-chaos expansion for certain applications where the boundedness of stochastic inputs is critical [2]. Thus, the truncated Gaussian distribution can be used to represent Gaussian-like inputs with no tails. Here we examine the performance of truncated Gaussian, although for random transport velocity the tails of the Gaussian assumption will not pose ill-posedness of the problem. The truncated Gaussian is obtained by a fifth-order Jacobi-chaos with $\alpha=\beta=10$ and will be denoted as $G^{(10,10)}$. In Figure 5, we show the first-, third-, and fifth-order approximation of $N(0,1)$. It can be seen that the target Gaussian distribution is closely approximated. However, $G^{(10,10)}$ has strictly bounded support. For more details on the construction of the truncated Gaussian distribution; see [28]. Here we employ this model to approximate the Gaussian input in the advection-diffusion problem from the previous section and use the corresponding Jacobi-chaos with $\alpha=\beta=10$ to solve the problem. The error convergence in the solution is shown in Figure 6, where we use the exact solution from Gaussian input. We can see that the error converges fast, and its rate is almost exponential with respect to polynomial order. For example, at polynomial order of $p=5$, the error in variance for Hermite-chaos is 0.1738 and for Jacobi-chaos is 0.0810 (see Figures 3 and 6 ). This indicates that the error in approximating the Gaussian input by $G^{(10,10)}$ is subdominant compared to the overall error.

In Figure 7, we show the PDF of the solution at its peak location at $t=\pi$. The solution of Jacobi-chaos approximates the exact PDF well, except the apparent Gibb's oscillations around zero. Note that here the PDF of the Jacobi-chaos solution is strictly bounded on both ends with no tails, consistent with the physics of the advection-diffusion problem.

The stochastic response at the solution peak is shown in Figure 8, along with the deterministic solution denoted by a dotted line. The stochastic solution is plotted using error bars, with a length of two standard deviations $(2 \sigma)$ around the mean 


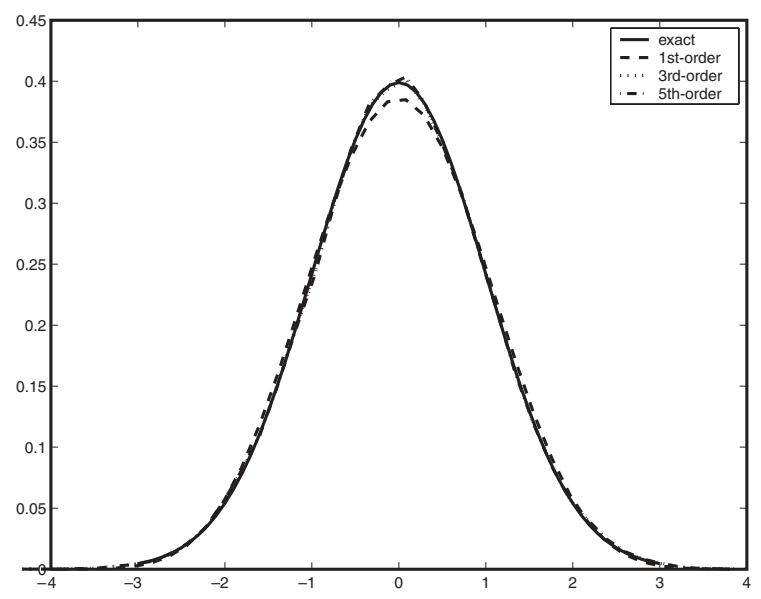

FIG. 5. $G^{(10,10)}$ approximation to Gaussian distribution $N(0,1)$.

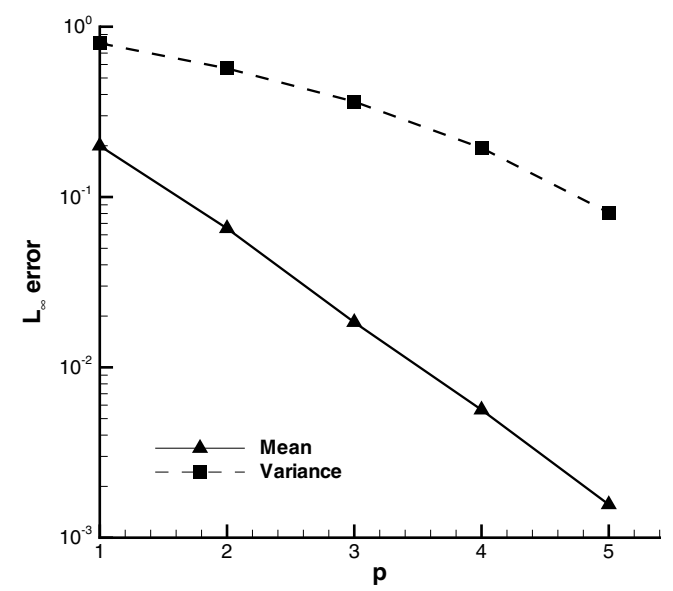

FiG. 6. $L^{\infty}$ error of Jacobi-chaos with truncated Gaussian $G^{(10,10)}$ random input at $T=3 \pi$.

values. The presence of the random perturbation in the transport velocity introduces extra diffusion in the mean solution, compared to the deterministic solution. It should be noted that for this particular type of random perturbation of (4.1), the stochastic effect disappears at $t=2 n \pi, n=0,1, \ldots$ (see exact solution (4.3)). This can be clearly seen from the error bars.

In Figure 9, the evolution of the mean solutions and variances for the truncated Gaussian input $G^{(10,10)}$ is shown at different times. The initial condition is a symmetric Gaussian-shape cone with circular contours. We observe that as the cone travels, it becomes asymmetric with elliptic contours due to the random perturbation introduced in the transport velocity of (4.1). After one revolution $(t=2 \pi)$, it returns to the symmetric shape as the random effect disappears at this instance. This is confirmed in Figure 9; the variance of the solution at $t=2 \pi$ is of the order $O\left(10^{-7}\right)$. The deformation of the solution contours resumes after this. The corresponding 


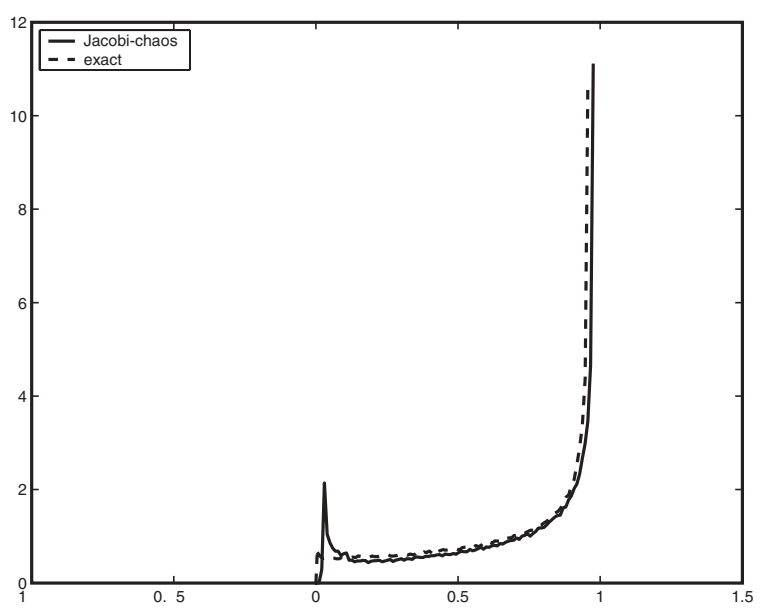

FIG. 7. PDF of the peak solution at $T=\pi$ with $G^{(10,10)}$ input and Jacobi-chaos expansion.

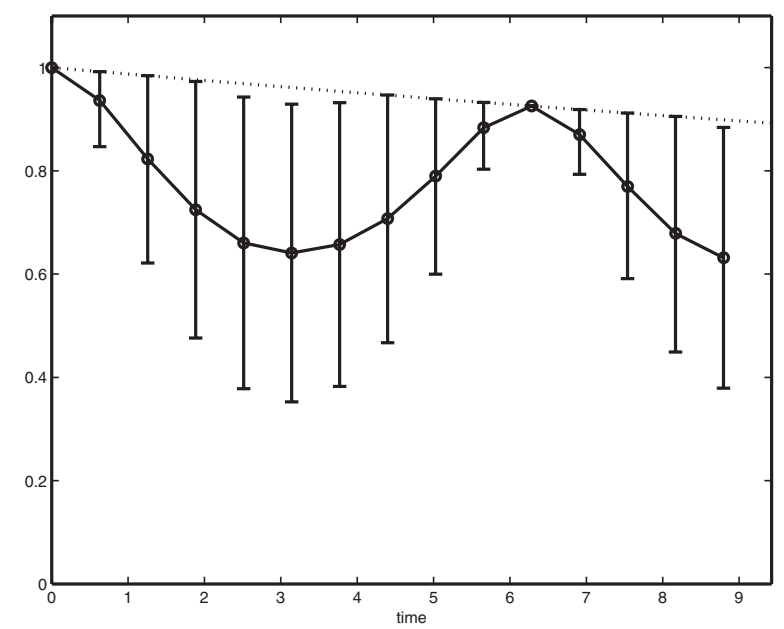

FIG. 8. Error bars of the evolution of the peak solution with $G^{(10,10)}$ input and Jacobi-chaos expansion. The circles represent the stochastic mean solution, and the dotted line represents the deterministic solution.

deterministic solution is free from such deformation, and thus the cone will retain its symmetric shape and simply decay over time.

4.2. Results with two-dimensional truncated Gaussian input. In this section we assume the random transport velocity takes the following form:

$$
\boldsymbol{u}=\left(y+0.05 y \xi_{1},-x-0.05 x \xi_{2}\right),
$$

where $\xi_{1}$ and $\xi_{2}$ are two independent Gaussian random variables with zero mean and unit variance. To avoid the unphysical tails in the solution, we use the truncated Gaussian $G^{(10,10)}$ to approximate $\xi_{1}$ and $\xi_{2}$, and employ the Jacobi-chaos to solve the equations.

The evolution of the stochastic response at the peak is shown in Figure 10, along with the deterministic solution denoted by a dotted line. The extra diffusion 


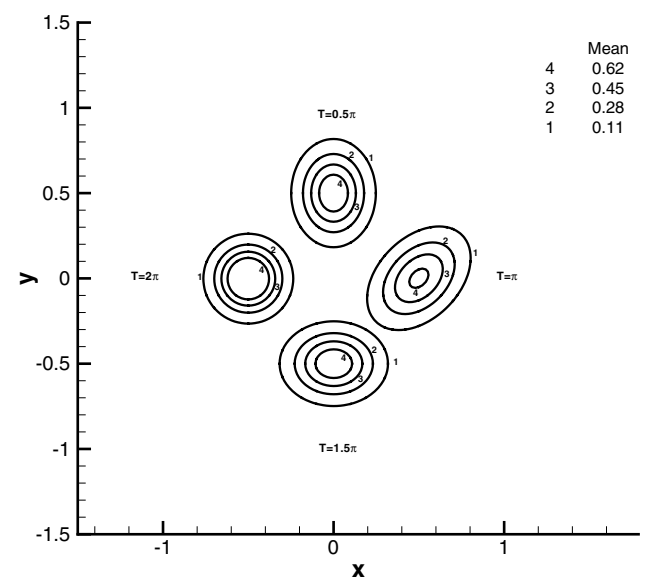

(a)

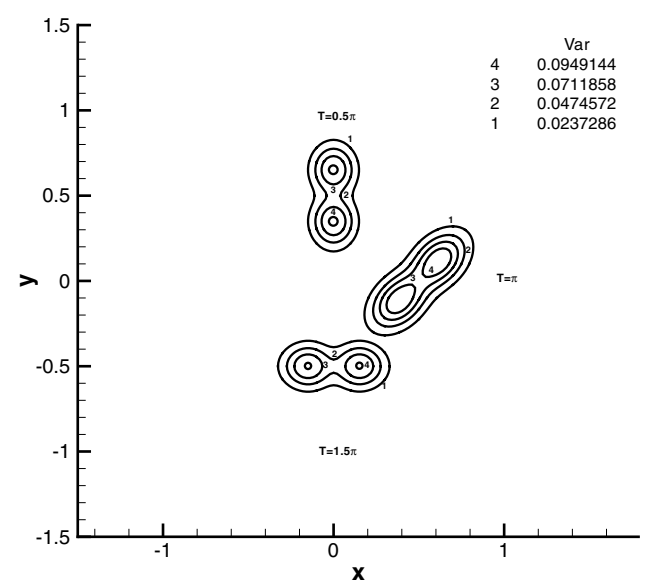

(b)

FIG. 9. Evolution of Jacobi-chaos solution with $G^{(10,10)}$ random input within $T \leq 2 \pi$. (a) Mean solution; (b) Variance. Note that at $t=2 \pi$ the variance is zero.

introduced by randomness in the transport velocity can be seen clearly, compared to the deterministic solution. As opposed to the example in the previous section, the random effect does not disappear after every $2 \pi$ evolution in time.

The evolution of the stochastic solutions at different times is plotted in Figure 11 (a) and (b) for the mean solutions and variances, respectively. Compared to the results of the one-dimensional truncated Gaussian perturbation in the previous section, the mean solution under the two-dimensional random perturbation deforms in a different way and does not return to the symmetric shape after each period $2 \pi$.

5. Conclusion. In this paper, the two-dimensional advection-diffusion equation with random transport velocity is studied. Such a problem is important not only 


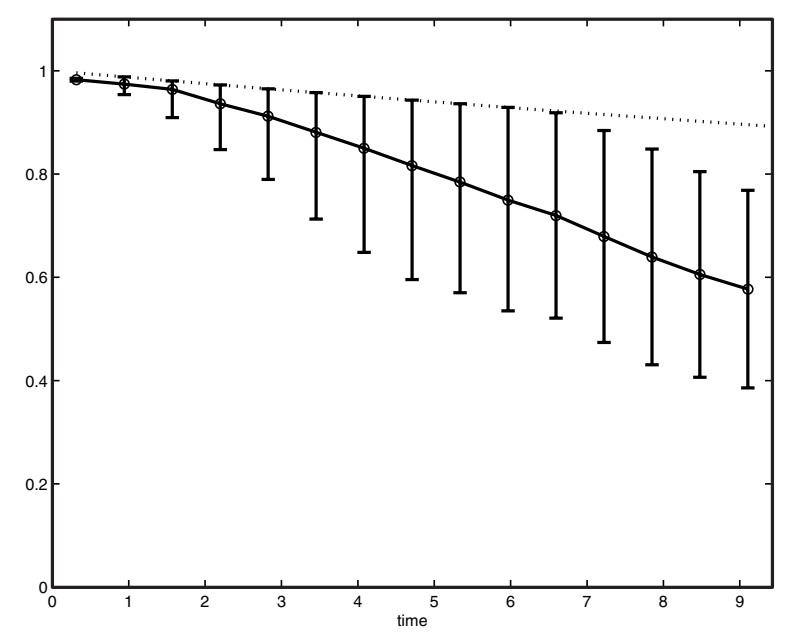

FIG. 10. Error bars of the evolution of the peak solution with two-dimensional "truncated" Gaussian input and Jacobi-chaos expansion. The circles represent the stochastic mean solution and the dotted line the deterministic solution.

from a numerical standpoint due to its time dependence and hyperbolic/parabolic character of the equation, but also from a physical standpoint, as it is a fundamental model in meteorology and other engineering applications.

While traditional studies on advection-diffusion equations focus on the deterministic aspects, we apply in this paper, for the first time, the generalized polynomial chaos expansion to the random advection-diffusion with uncertain inputs. In particular, we focus on the Hermite-chaos and Jacobi-chaos, corresponding to Gaussian random inputs and beta random inputs, respectively. An exact solution, which offers a testbed for the new formulation, is presented, and we examined the convergence of the generalized polynomial chaos expansion.

A truncated Gaussian model, based on Jacobi-chaos, is employed to approximate a Gaussian input and produce solutions with bounded support. In some applications, the true solution has bounded support, and it is preferred that the numerical solution retains the same feature. This is an issue that could have numerical and physical implications, e.g., stability of a numerical scheme, dispersion of species in turbulent flows, etc. In applications where the bounded support, either in the stochastic inputs or in the stochastic outputs, is important, the Jacobi-chaos expansion can be considered as an effective approach to achieve this goal. 


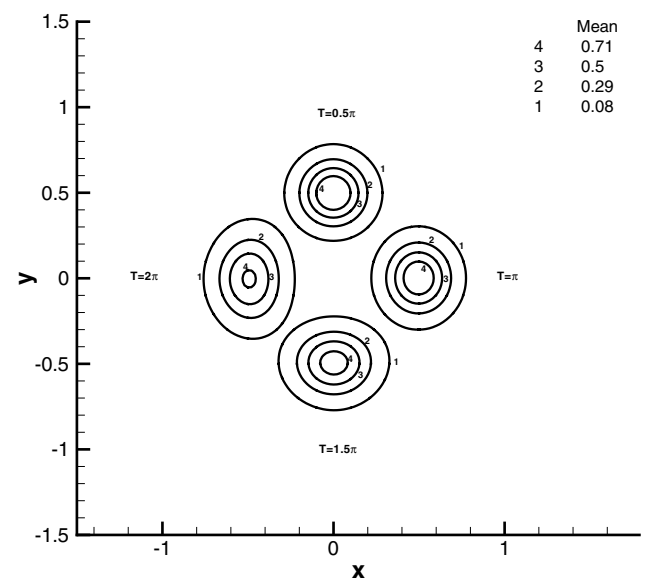

(a)

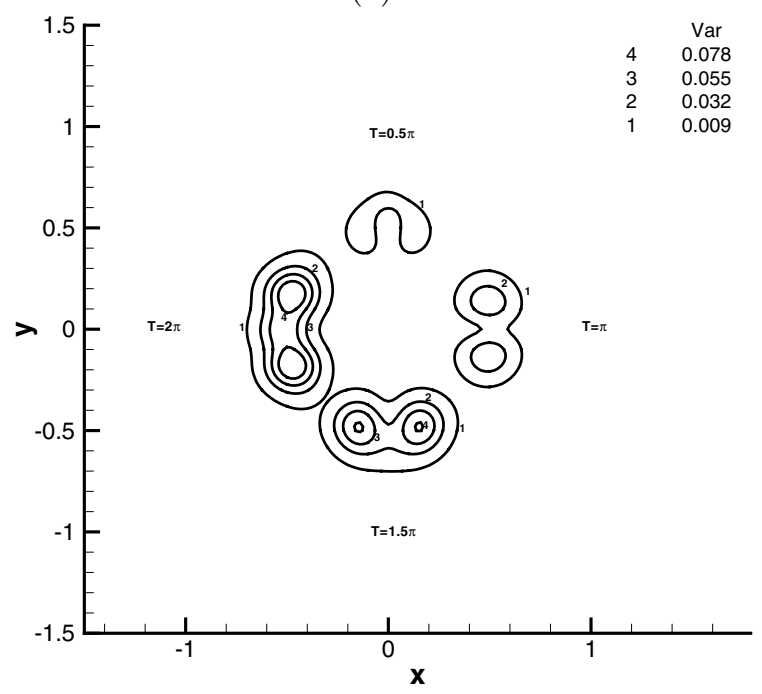

(b)

FIG. 11. Evolution of Jacobi-chaos solution with two-dimensional-Gaussian random input within $T \leq 2 \pi$. (a) Mean solution; (b) variance. 


\section{REFERENCES}

[1] R. Askey and J. Wilson, Some Basic Hypergeometric Polynomials That Generalize Jacobi Polynomials, Mem. Amer. Math. Soc. 319, AMS, Providence RI, 1985.

[2] I. BABUŠKKA AND P. Chatzipantelidis, On solving elliptic stochastic partial differential equations, Comput. Methods Appl. Mech. Engrg., 191 (2002), pp. 4093-4122.

[3] R. Cameron And W. Martin, The orthogonal development of nonlinear functionals in series of Fourier-Hermite functionals, Ann. Math., 48 (1947), pp. 385-392.

[4] A. Chorin, Hermite expansion in Monte-Carlo simulations, J. Comput. Phys., 8 (1971), pp. $472-482$.

[5] M. Deb, I. BabušKa, And J. Oden, Solution of stochastic partial differential equations using Galerkin finite element techniques, Comput. Methods Appl. Mech. Engrg., 190 (2001), pp. 6359-6372.

[6] C. Gear, Numerical Initial Value Problems in Ordinary Differential Equations, Prentice-Hall, Englewood Cliffs, NJ, 1971.

[7] R. Ghanem, Scales of fluctuation and the propagation of uncertainty in random porous media, Water Resourc. Res., 34 (1998), pp. 2123-2126.

[8] R. GHAnEM, Ingredients for a general purpose stochastic finite element formulation, Comput. Methods Appl. Mech. Engrg., 168 (1999), pp. 19-34.

[9] R. Ghanem And J. Red-Horse, Propagation of uncertainty in complex physical systems using a stochastic finite elements approach, Phys. D, 133 (1999), pp. 137-144.

[10] R. Ghanem and P. Spanos, Stochastic Finite Elements: A Spectral Approach, Springer-Verlag, New York, 1991.

[11] J. Glimm And D. Sharp, Stochastic partial differential equations: Selected applications in continuum physics, in Stochastic Partial Differential Equations: Six Perspectives, R. Carmona and B. Rozovskii, eds., AMS, Providence, RI, 1999, pp. 3-44.

[12] G. Karniadakis and S. Sherwin, Spectral/hp Element Methods for CFD, Oxford University Press, New York, 1999.

[13] M. Kleiber And T. Hien, The Stochastic Finite Element Method, John Wiley \& Sons Ltd., Chichester, UK, 1992.

[14] R. Koekoek and R. Swarttouw, The Askey-Scheme of Hypergeometric Orthogonal Polynomials and Its q-Analogue, Tech. Report 98-17, Department of Technical Mathematics and Informatics, Delft University of Technology, Delft, The Netherlands, 1998.

[15] O. Le Maitre, O. Knio, H. Najm, and R. Ghanem, A stochastic projection method for fluid flow: Basic formulation, J. Comput. Phys., 173 (2001), pp. 481-511.

[16] M. LoÈve, Probability Theory, 4th ed., Springer-Verlag, New York, 1977.

[17] H. Matthies and C. Bucher, Finite elements for stochastic media problems, Comput. Methods Appl. Mech. Engrg., 168 (1999), pp. 3-17.

[18] W. Oberkampf, T. Trucano, and C. Hirsch, Verification, Validation, and Predictive Capability in Computational Engineering and Physics, Tech. Report SAND2003-3769, Sandia National Laboratories, Albuquerque, NM, 2003.

[19] H. OGura, Orthogonal functionals of the Poisson process, IEEE Trans. Information Theory, IT-18 (1972), pp. 473-481.

[20] S. Orszag and L. Bissonnette, Dynamical properties of truncated Wiener-Hermite expansions, Phys. Fluids, 10 (1967), pp. 2603-2613.

[21] G. Papanicolaou, Diffusion in random media, in Surveys in Applied Mathematics, J. Keller, D. McLaughlin, and G. Papanicolaou, eds., Plenum Press, New York, 1995, pp. 205-255.

[22] W. Schoutens, Stochastic Processes in the Askey Scheme, Ph.D. thesis, K.U. Leuven, Leuven, Belgium, 1999.

[23] N. WiEner, The homogeneous chaos, Amer. J. Math., 60 (1938), pp. 897-936.

[24] C. Winter and D. Tartakovsky, Mean flow in composite porous media, Geophys. Res. Lett., 27 (2000), pp. 1759-1762.

[25] D. XIU AND G. KARNIADAKIS, Modeling uncertainty in steady state diffusion problems via generalized polynomial chaos, Comput. Methods Appl. Math. Engrg., 191 (2002), pp. 49274948.

[26] D. XIU AND G. E. KARNIADAKIS, The Wiener-Askey polynomial chaos for stochastic differential equations, SIAM J. Sci. Comput., 24 (2002), pp. 619-644.

[27] D. Xiu And G. Karniadakis, Modeling uncertainty in flow simulations via generalized polynomial chaos, J. Comput. Phys., 187 (2003), pp. 137-167.

[28] D. Xiu And G. Karniadakis, Supersensitivity due to uncertain boundary conditions, Internat. J. Numer. Methods Engrg., to appear. 